\title{
Hematolymphoid Neoplasms in Serous Effusions: Morphological Spectrum, Distribution, and Role of Ancillary Techniques-A Retrospective Analysis of 75 cases
}

\author{
Renu Sukumaran ${ }^{1}$ Nileena Nayak ${ }^{1}$ Rekha A. Nair \\ Geetha Narayanan ${ }^{2}$ Priyakumari Thankamony ${ }^{3}$ \\ ${ }^{1}$ Department of Pathology, Regional Cancer Centre, \\ Thiruvananthapuram, Kerala, India \\ 2 Department of Medical Oncology, Regional Cancer, Centre, \\ Thiruvananthapuram, Kerala, India \\ ${ }^{3}$ Department of Paediatric Oncology, Regional Cancer Centre, \\ Thiruvananthapuram, Kerala, India \\ Ind J Med Paediatr Oncol 2021;42:451-456.
}

\author{
Address for correspondence Renu Sukumaran, MD, Regional Cancer \\ Centre, Thiruvananthapuram, Kerala 695011, India \\ (e-mail: renu.sukumaran@gmail.com).
}

\begin{abstract}
Introduction Involvement of body fluids can occur at the time of diagnosis or during the disease course of hematolymphoid neoplasms. Cytodiagnosis of malignant effusion is important in effective clinical management.

Objectives (1) The aims of the study were to determine the frequency of distribution of various hematolymphoid neoplasms involving body fluids, (2) to study the morphology of hematolymphoid neoplasms in fluids, and (3) to assess the role of ancillary techniques in the diagnosis.

Materials and Methods In this retrospective study, all cases of hematolymphoid neoplasms involving body fluids diagnosed from January 2016 to December 2018 were evaluated.

Results During the 3-year period, there were 75 cases of hematological malignancies involving body fluids. These included 48 male patients and 27 female patients. Pleural fluid was involved in majority of cases (56 cases; $74.67 \%$ ), followed by ascitic fluid (17 cases; $22.67 \%$ ), and pericardial fluid ( 2 cases; $2.67 \%$ ). High cellularity, monotonous population of cells, high nuclear-cytoplasmic (N/C) ratio, indentation/irregularity of nuclear membrane, immature chromatin/irregular clumping of chromatin, increased mitosis, and karyorrhexis were the key features which helped to differentiate between reactive and neoplastic processes. There were 35 cases of B-cell neoplasms, 33 cases of T-cell neoplasms, and seven cases of myeloid neoplasms involving body cavity fluids. T-lymphoblastic lymphoma was the most common subtype (29 cases; $38.7 \%$ ), followed by diffuse large B-cell lymphoma (DLBCL) (12 cases; 16\%). In 53 cases, effusion was present in the initial presentation itself. Initial

Keywords

- Effusion cytology

- hematolymphoid neoplasm

- peritoneal fluid

- pleural fluid diagnosis was made in effusion cytology in 25 cases (33.33\% of the total), with the help of flow cytometry in 20 cases, and immunohistochemistry $(\mathrm{IHC})$ in cell blocks in five cases.

Conclusion Diagnosis of hematolymphoid neoplasms in body fluids based on correlation with clinical details, critical evaluation of cytology findings, and comparison with previous diagnosis along with the judicious use of ancillary techniques helps in deciding an early treatment plan.
\end{abstract}

DOI https://doi.org/ 10.1055/s-0041-1731844. ISSN 0971-5851. (c) 2021. Indian Society of Medical and Paediatric Oncology. All rights reserved.

This is an open access article published by Thieme under the terms of the Creative Commons Attribution-NonDerivative-NonCommercial-License, permitting copying and reproduction so long as the original work is given appropriate credit. Contents may not be used for commercial purposes, or adapted, remixed, transformed or built upon. (https://creativecommons.org/ licenses/by-nc-nd/4.0/)

Thieme Medical and Scientific Publishers Pvt. Ltd., A-12, 2nd Floor, Sector 2, Noida-201301 UP, India 


\section{Introduction}

Effusions indicate a pathologic process. The etiology of effusions includes neoplastic and nonneoplastic conditions. Pathogenesis of effusion in hematologic malignancy is multifactorial. Infection due to impaired immune system, impaired lymphatic drainage due to lymph node enlargement, venous obstruction or increase in vascular permeability, and radiation-associated changes can lead to body cavity effusions. ${ }^{1}$ The presence of malignant cells in effusions is a sign of advanced and generalized disease. Hematolymphoid neoplasms can involve body cavity fluids at the time of diagnosis or during the course of the disease. The frequency of fluid involvement varies depending on the subtypes. ${ }^{2}$ In cytology smears, the differential diagnoses include lymphocyte-rich/plasma cell-rich reactive effusions, round cell tumors, and carcinomas. Cytodiagnosis of malignant effusion is important in prompt effective clinical management.

\section{Materials and Methods}

In this retrospective study, 75 cases of hematolymphoid neoplasms involving body cavity fluids diagnosed from January 2016 to December 2018 were analyzed.

The frequency of involvement of different subtypes and the distribution in different body cavities were analyzed. The cytomorphology of hematolymphoid neoplasms in effusion, including cellularity, cell arrangement, cell size, cytoplasmic details (amount of cytoplasm and cytoplasmic vacuolations), nuclear-to-cytoplasmic ratio, nuclear membrane (regular, irregular, and indentation), chromatin (fine, coarse, and immature), nucleoli (not prominent/prominent, single/ multiple), mitosis, karyorrhexis, and necrosis was studied.

In the cases with a prior diagnosis and subtyping of hematolymphoid neoplasm on biopsy material, blood smears, or marrow, the morphology of cells in fluids was compared and matched with that of the initial diagnostic samples. Ancillary techniques-flow cytometric (FC) analysis of fluids and immunohistochemistry (IHC) in cell blockswere done in the cases showing effusion as the predominant finding, those cases with morphological features suggestive of hematolymphoid neoplasms, and those cases without a prior diagnosis. In this study, the role of ancillary techniques in the diagnosis and subtyping was evaluated.

The study methodology was approved by the Scientific Review Committee of the Regional Cancer Centre on October 22, 2019, IRB No:10/2019/14. Waiver of consent was obtained as the study was retrospective in nature. The procedures followed were in accordance with the ethical standards of the responsible committee on human experimentation and with the Helsinki Declaration of 1964, as revised in 2013.

\section{Results}

During the 3-year period, there were 75 cases of hematolymphoid neoplasms involving body fluids. These included 48 male patients and 27 female patients. Fifty-two were adults and 23 were pediatric patients.

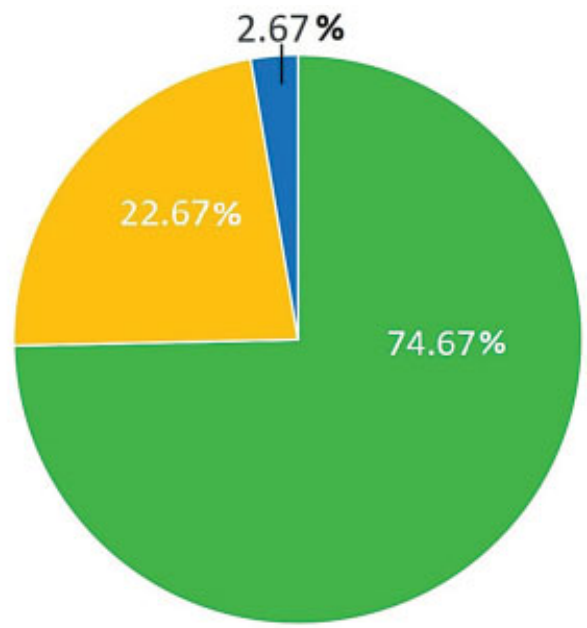

\section{a Pleural fluid $=$ Ascitic fluid $\quad$ Pericardial fluid}

Fig. 1 Distribution of hematolymphoid neoplasms in body fluids.

Pleural fluid was involved in majority of cases (56 cases; $74.67 \%$ ), followed by ascitic fluid (17 cases; $22.67 \%$ ), and pericardial fluid (two cases; $2.67 \%$ ) (-Fig. 1).

There were 35 cases of B-cell neoplasms, 33 cases of T-cell neoplasms, and seven cases of myeloid neoplasms involving

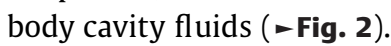

High cellularity, monotonous population of cells, high nuclear-cytoplasmic $(\mathrm{N} / \mathrm{C})$ ratio, indentation/irregularity of nuclear membrane, immature chromatin/irregular clumping of chromatin, increased mitosis, and karyorrhexis were the key features which helped to differentiate between reactive and neoplastic processes.

B-cell neoplasms included diffuse large B-cell lymphoma (DLBCL) (12 cases), plasma cell myeloma (9 cases), Burkitt lymphoma ( 8 cases), follicular lymphoma ( 4 cases), hairy cell leukemia (1 case), and plasmablastic lymphoma (1 case).

In DLBCL, the smears were cellular with medium- to largesized cells with a moderate amount of cytoplasm, large nucleus, coarse chromatin, and one or more nucleoli. Mitoses and karyorrhectic debris were also noted.

Highly cellular smears showing medium-sized cells with high $\mathrm{N} / \mathrm{C}$, irregular clumping of chromatin, multiple small

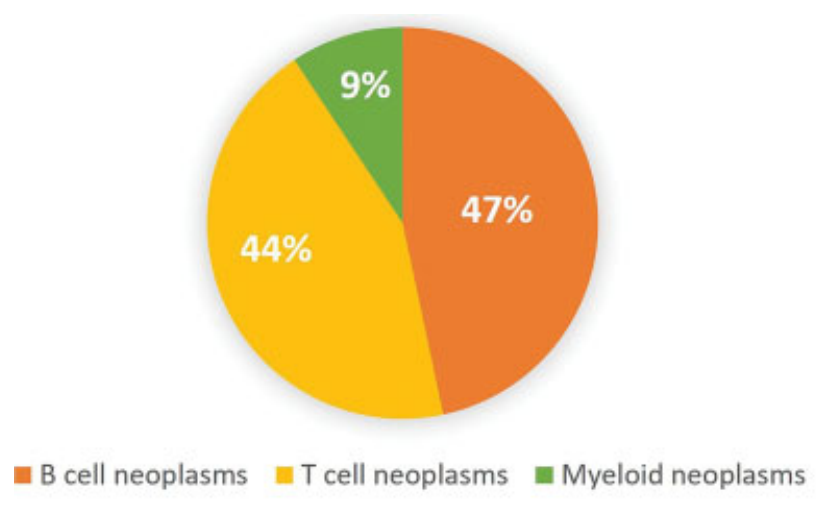

Fig. 2 Subclassification of hematolymphoid neoplasms in fluids. 
nucleoli, brisk mitosis, and karyorrhectic debris were the important features leading to the diagnosis of Burkitt lymphoma.

In hairy cell leukemia and plasmablastic lymphoma, the effusion was present in the disease relapse. Correlation of cytomorphologic features with that of the original diagnostic material aided in the diagnosis.

T-cell neoplasms included T-lymphoblastic acute leukemia/lymphoma (T ALL), anaplastic large cell lymphoma (ALCL), and adult T-cell leukemia/lymphoma (ATLL). Of the 29 cases of T ALL, 24 cases showed effusion in the initial presentation and 5 cases during the course of the disease. Cases of T ALL showed highly cellular smears with a monotonous population of intermediate-sized cells along with immature chromatin, inconspicuous nucleoli, and scant cytoplasm. Frequent mitosis and karyorrhexis were also noted. Of the 24 cases of T ALL, with effusion in the initial presentation, 14 cases were diagnosed on FC evaluation of effusion fluids. There were three cases of ATLL. Effusion was present in the initial presentation in one case and during disease relapse in two cases. The typical convoluted/flower cells helped in the morphological diagnosis of ATLL. FC evaluation was done in effusion fluid in one case, with initial presentation as lymphadenopathy and pleural effusion. In ALCL, the initial manifestation was ascites. Effusion cytology showed singly scattered large, atypical cells, some with wreath-like nuclei. IHC on cell block preparation showed the atypical cells to be positive for $\mathrm{CD} 3, \mathrm{CD} 30$, and anaplastic lymphoma kinase.

Myeloid neoplasms included acute myeloid leukemia (AML) and myeloid blast crisis of chronic myeloid leukemia (CML). There were four cases of AMLM4/M5, one case of AML M0, one case of AML M1, and one case of blast crisis of CML. One case of AML M4/M5 was initially diagnosed in pleural effusion with the help of FC. In all the other cases, the morphology of atypical cells in effusions was compared and correlated with a previous diagnosis of myeloid neoplasms in peripheral blood or bone marrow.

T ALL was the most common subtype of hematolymphoid neoplasm involving body cavity fluids (29 cases; 38.7\%), followed by DLBCL (12 cases; $16 \%$ ).

In 53 cases, effusion was present in the initial presentation itself. Initial diagnosis of hematolymphoid neoplasm was made in effusion cytology in 25 cases $333.33 \%$ of the total), with the help of FC in 20 cases, and IHC in cell blocks in 5 cases (-Figs 3-8).

FC diagnosis of T ALL was made in 14 cases, Burkitt lymphoma in 4 cases, AML in 1 case, and ATLL in 1 case. IHC in cell block preparation helped in making the diagnosis of DLBCL in three cases, T ALL in one case, and ALCL in one case.

\section{Discussion}

Effusions can occur in a wide spectrum of diseases. It includes neoplastic and nonneoplastic conditions. The nonneoplastic causes include posttraumatic, inflammatory (infection or connective tissue disorders), and circulatory

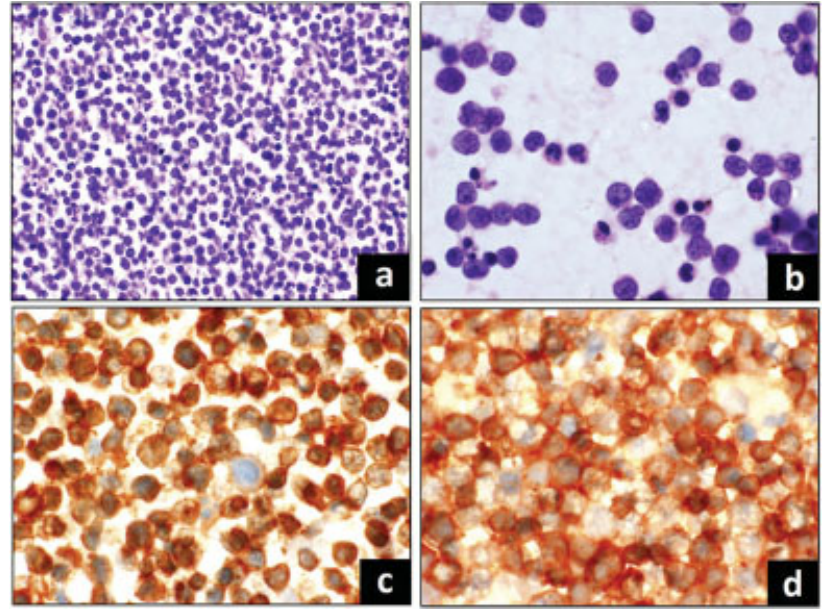

Fig. 3 T-lymphoblastic lymphoma involving pleural fluid. (a) Highly cellular smear, (b) singly dispersed cells with irregular nuclear membrane, immature chromatin, (c) CD3 positive, (d) CD10 positive.

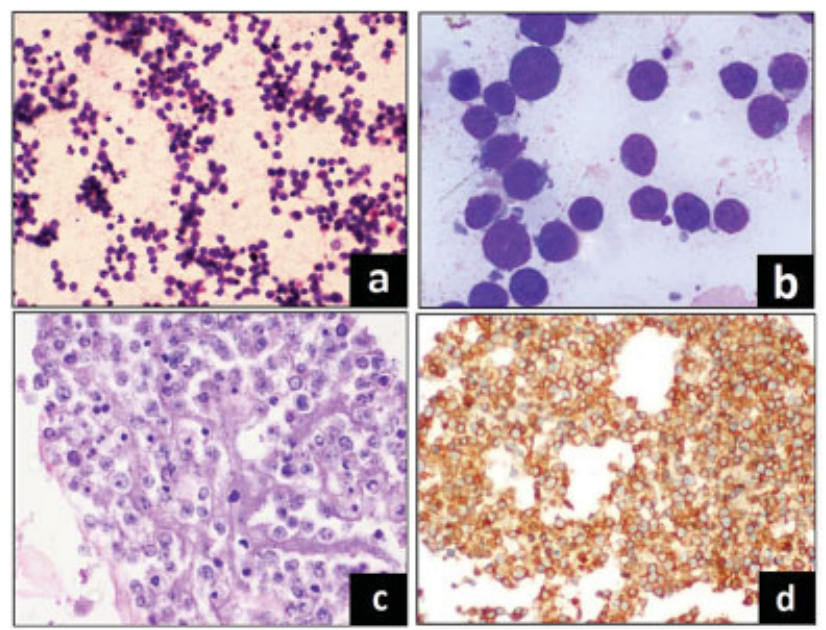

Fig. 4 Diffuse large B-cell lymphoma involving ascitic fluid. (a) Cellular smear, (b) large, atypical cells, (c) cell block preparation, (d) CD20 positive.

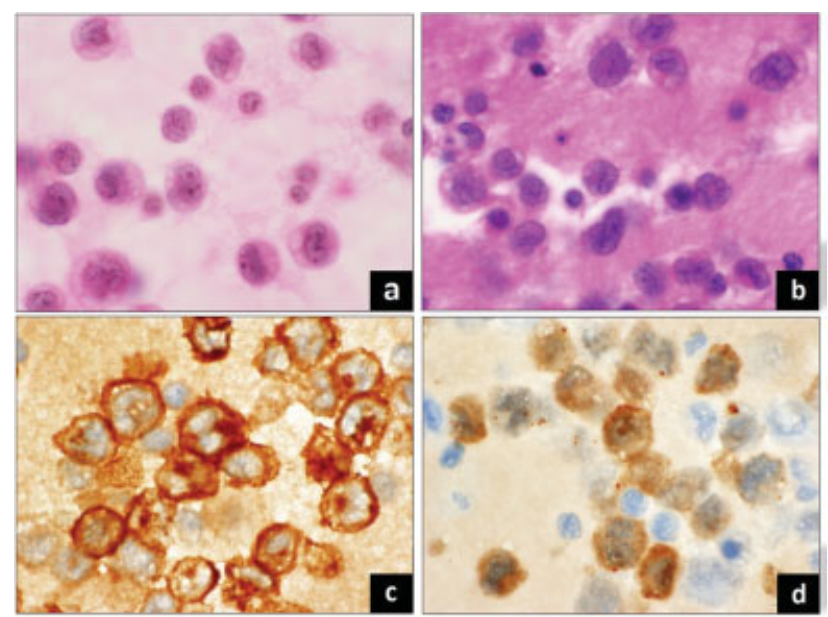

Fig. 5 Anaplastic large cell lymphoma involving ascitic fluid. (a) Smear showing large, atypical cells with irregular nuclei, (b) cell block preparation, (c) CD30 positive, (d) ALK positive. 

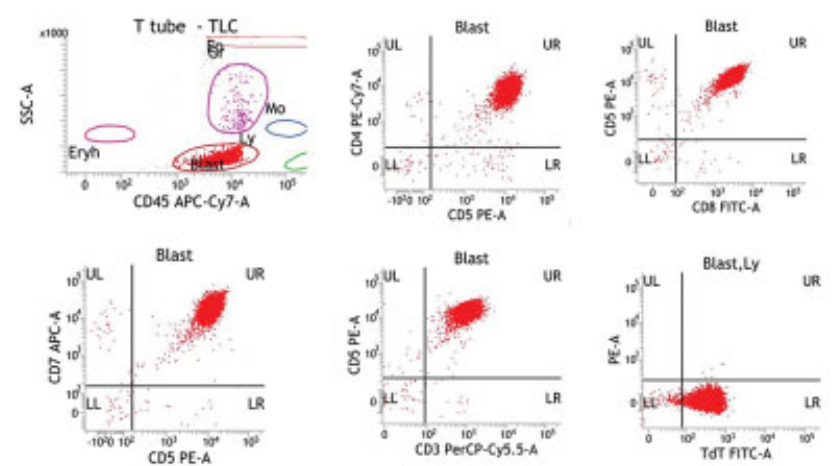

Fig. 6 T-lymphoblastic lymphoma pleural fluid, flow cytometry (FC). Gated population of cells are CD5 positive, CD4 positive, CD8 positive, CD7 positive, $\mathrm{CD} 3$ positive, and Tdt positive.
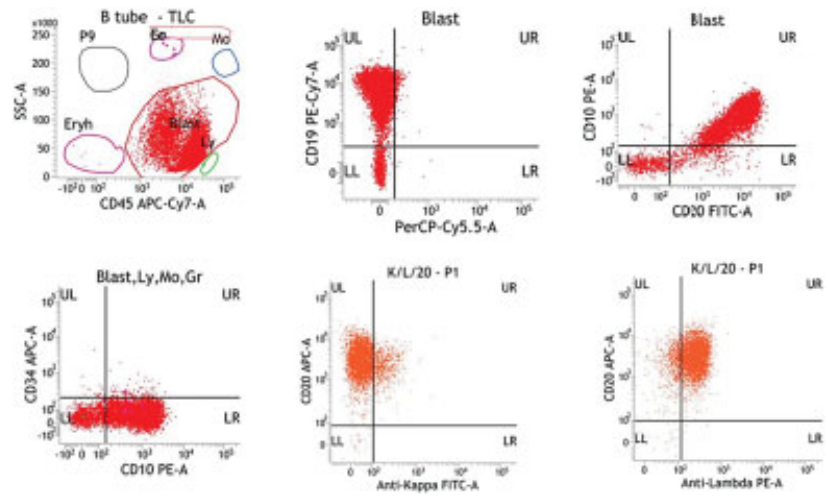

Fig. 7 Burkitt lymphoma ascitic fluid, flow cytometry (FC). Gated population of cells are CD19 positive, CD20 positive, CD10 positive, CD34 negative and show lambda light chain restriction.

(congestive heart failure and lymphatic obstruction). Dysfunction of liver or kidney can also result in the development of effusions. Effusion can be the presenting symptom or can occur in the course of various epithelial and hematopoietic malignancies. $^{1-3}$

\section{Effusion in Hematolymphoid Neoplasms}

The pathogenesis of effusion in hematologic malignancy is multifactorial. Infection due to impaired immune system is the most common cause. Impaired lymphatic drainage due to lymph node enlargement, venous obstruction, or increase in vascular permeability can result in effusion. Radiation-associated changes and infiltration of the serosal membranes by the tumor can lead to the development of effusion.

Malignant pleural effusions are common in patients with hematologic malignancies, while involvement of the peritoneal and pericardial cavities seems to be less common. Up to $10 \%$ of malignant pleural effusions with a positive cytologic examination are due to non-Hodgkin lymphoma (NHL). ${ }^{3,4}$ Gilbert et al noted that among 91 patients with hematologic disease requiring placement of an indwelling pleural catheter, $62 \%$ had a diagnosis of a lymphoma, $21 \%$ had leukemia, and $13 \%$ had multiple myeloma. ${ }^{4,5}$ The presence of cells of a hematologic malignancy in effusion indicates advanced and generalized disease.
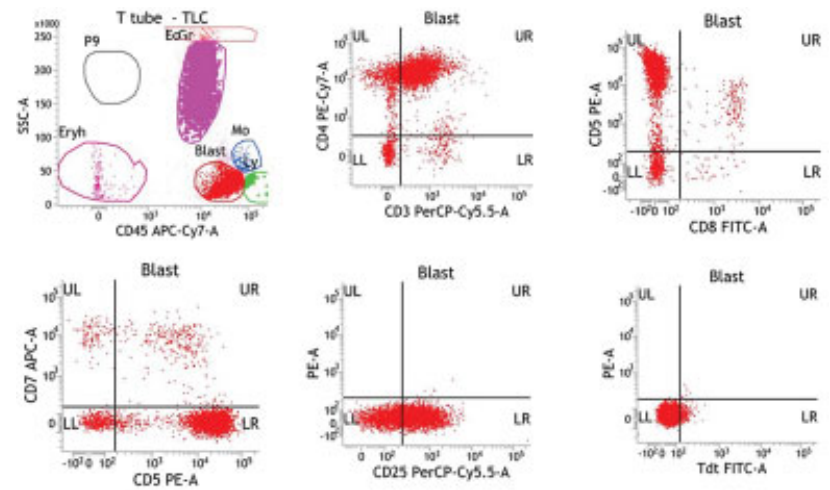

Fig. 8 Adult T-cell leukemia/lymphoma pleural fluid, flow cytometry (FC). Gated population of cells are CD3 positive, CD4 positive, CD5 positive, CD25 positive, CD7 negative, CD8 negative, and Tdt negative.

\section{Cytodiagnosis of Hematolymphoid Neoplasms in Effusion Fluids}

Diagnosis of hematological malignancies can be challenging in effusion cytology. Cytopathologists frequently encounter lymphocyte-rich effusions. The common causes of lymphocyte-rich effusions are tuberculosis, fungal infections, and connective tissue disorders. Cells of low-grade NHL usually have a deceptively bland appearance. In contrast, reactive cells in lymphocyte-rich effusions may display striking atypia. Large cell lymphomas must be differentiated from epithelial malignancies. Other round cell neoplasms, mesothelioma, and metastatic germ cell tumors should also be kept in the differential diagnosis. ${ }^{2,6}$ Diagnostic confusion is more when malignant effusion occurs as the initial clinical presentation. Cytomorphologic evaluation along with IHC in the cell block and FC immunophenotyping of effusion fluids yield valuable information in the differential diagnosis of lymphocyte-rich infiltrates. ${ }^{2,7}$

\section{Flow Cytometry in Effusions}

Effusion fluids contain suspensions with viable cells with well-preserved surface antigens. Surface antigenicity of individual cells identified by FC will help to precisely subclassify these cells. ${ }^{4,8,9}$

All lymphocyte-rich effusions should be thoroughly evaluated by cytopathologists. This helps to triage the samples. The samples which contain inflammatory cells or nonhematopoietic malignancy should be excluded from FC. Knowledge of the typical pitfalls of FC in the diagnosis of hematopoietic neoplasms in effusion fluids is essential to avoid false-positive and false-negative results. ${ }^{4,9}$ The lymphocytic population of reactive effusions consists practically exclusively of T-cell lymphocytes, and the large CD3-positive population is not indicative of a T-cell lymphoma. The awareness of this fact will help to avoid false-positive diagnosis. ${ }^{4}$ One should be aware of the risk of false-negative results in the cases of large B-cell lymphomas due to extensive necrosis or mechanical destruction of fragile cytoplasmic borders of tumor cells, resulting in artificial loss of surface antigens. In such cases, correlation with conventional 
microscopic findings is crucial. Another pitfall is the taprelated contamination of body cavity fluids by blood. This is especially important in patients with chronic lymphocytic leukemia, which may lead to the appearance of a small neoplastic cell population in the FC of effusion samples, resulting in false-positive results for the fluid. ${ }^{4}$

In the current study, FC diagnosis of T ALL was made in 14 cases, Burkitt lymphoma in four cases, AML in one case, and ATLL in one case.

\section{Cell block Preparation in Effusion}

Cell block preparation is important especially in cases where there is limited availability of other diagnostic material. In our series, IHC in cell block preparation helped in making the diagnosis of DLBCL in three cases, T ALL in one case, and ALCL in one case. The panel of markers was selected according to the morphological details of cells.

\section{Effusion in T-cell non-Hodgkin lymphoma}

T-cell NHLs are more frequently associated with effusions than B-cell NHLs. ${ }^{6,7,10}$ In their study, Das et al observed pleural effusions in $41.6 \%$ of T-lymphoblastic lymphomas and in $3.8 \%$ of cases in other NHL types. Of the 24 cases in their series, Monappa et al observed nine cases of T-cell NHL involving fluids. ${ }^{11}$ Chen et al studied cases with pleural effusion as an initial clinical presentation of T ALL and opined that the diagnosis of T-cell lymphoblastic leukemia/lymphoma should be considered in young patients with mediastinal mass, with pleural effusion showing predominant small- to medium-sized lymphoid cells with round-to-oval convoluted nuclei and fine chromatin with frequent mitosis and karyorrhexis. ${ }^{7}$ The present study showed 33 cases of T-cell neoplasms, which included 29 cases of T ALL, one case of ALCL, and three cases of ATLL.

\section{Effusions in B-cell non-Hodgkin Lymphoma}

Of the 35 cases of B-cell NHL involving fluids in the present study, 12 were DLBCL. Serous effusions are a common manifestation of DLBCL. Monappa et al, in their series of 24 cases of hematolymphoid neoplasms in effusion cytology, observed nine cases of DLBCL involving effusion fluids. ${ }^{11}$ Chen et al observed that $18.7 \%$ of patients with DLBCL had cytologically examined serous effusions during the course of their disease. In $58.5 \%$ of these cases, effusion was present at the time of the first diagnosis. Forty-six percent of these effusions were cytologically diagnosed as malignant. These authors observed that malignant effusion was a poor prognostic indicator and was worse for the patients having malignant effusion at presentation. ${ }^{8,12}$

Burkitt lymphoma is an aggressive lymphoma with medium-sized monomorphic cells with high mitosis. Effusions are common in Burkitt lymphoma and can occur in up to $60 \%$ of pleural fluids and $90 \%$ of ascitic fluids in sporadic Burkitt lymphoma.

Follicular lymphoma is one of the common lymphoma types. Malignant effusions are usually seen during the disease relapse.
Studies have shown that around 6\% of cases of plasma cell myeloma can develop body cavity effusions. In most of the cases, the effusions are nonneoplastic. Congestive heart disease, renal insufficiency, liver dysfunction, inflammation, or infection can result in nonmyelomatous effusion. Rarely (in $<1 \%$ of cases), a myelomatous infiltration of serous membranes may be the cause of fluid accumulation. ${ }^{13}$ In our study, there were nine cases of plasma cell myeloma involving fluids, and all these cases were during disease relapse.

\section{Effusion in Myeloid Neoplasms}

The etiology of effusion in AML and CML includes bacterial or viral infections and complications of chemotherapy. Leukemic effusions are rare in myeloid neoplasms. The possible mechanisms include extramedullary proliferation of quiescent leukemic clone or subclinical marrow relapse, with seeding to extramedullary sites. ${ }^{14,15}$ In myeloproliferative neoplasms, effusions due to disease infiltration are mostly reported in advanced stages, shortly before blastic transformation.

\section{Conclusion}

Hematolymphoid neoplasms involving body cavity fluids indicate advanced and generalized disease. The accuracy in the diagnosis of hematolymphoid neoplasms in effusion cytology depends on critical evaluation of morphologic details, correlation with clinical details, and comparison with previous diagnosis along with judicious use of ancillary techniques. Cytodiagnosis of hematolymphoid neoplasms can provide an early breakthrough in patient management. These diagnostic perspectives stress the important role of cytopathologists in the integrative diagnostic process.

Presentation at a Meeting

APCON 2019.

Source of funding

Nil.

Conflict of Interest

None declared.

\section{References}

1 Elis A, Blickstein D, Mulchanov I, et al. Pleural effusion in patients with non-Hodgkin's lymphoma: a case-controlled study. Cancer 1998;83(08):1607-1611

2 Alexandrakis MG, Passam FH, Kyriakou DS, Bouros D. Pleural effusions in hematologic malignancies. Chest 2004;125(04): 1546-1555

3 Vakil E, Jimenez CA, Faiz SA. Pleural effusions in hematologic malignancies and their management with indwelling pleural catheters. Curr Opin Pulm Med 2018;24(04):384-391

4 Bode-Lesniewska B. Flow cytometry and effusions in lymphoproliferative processes and other hematologic neoplasias. Acta Cytol 2016;60(04):354-364

5 Gilbert CR, Lee HJ, Skalski JH, et al. The use of indwelling tunnelled pleural catheters for recurrent pleural effusions in patients with 
hematologic malignancies: A multicentre study. Chest 2015;148 (03):752-758

6 Bangerter M, Hildebrand A, Griesshammer M. Combined cytomorphologic and immunophenotypic analysis in the diagnostic workup of lymphomatous effusions. Acta Cytol 2001;45(03):307-312

7 Chen L, Zhang JS, Cui D, Liu DG. Cytological diagnosis of T lymphoblastic leukemia/lymphoma in patients with pleural effusion as an initial clinical presentation: Two case reports of an algorithmic approach using complete immunohistochemical phenotyping. Acta Cytol 2015;59(06):485-492

8 Palmer HE, Wilson CS, Bardales RH. Cytology and flow cytometry of malignant effusions of multiple myeloma. Diagn Cytopathol 2000;22(03):147-151

9 Yu GH, Vergara N, Moore EM, King RL. Use of flow cytometry in the diagnosis of lymphoproliferative disorders in fluid specimens. Diagn Cytopathol 2014;42(08):664-670
10 Das DK. Serous effusions in malignant lymphomas: a review. Diagn Cytopathol 2006;34(05):335-347

11 Monappa V, Reddy SM, Kudva R. Hematolymphoid neoplasms in effusion cytology. Cytojournal 2018;15; 15

12 Chen YP, Huang HY, Lin KP, Medeiros LJ, Chen TY, Chang KC. Malignant effusions correlate with poorer prognosis in patients with diffuse large B-cell lymphoma. Am J Clin Pathol 2015;143 (05):707-715

13 Deshpande AH, Munshi MM. Pleural effusion as an initial manifestation of multiple myeloma. Acta Cytol 2000;44(01):103-104

14 Faiz SA, Bashoura L, Lei X, et al. Pleural effusions in patients with acute leukemia and myelodysplastic syndrome. Leuk Lymphoma 2013;54(02):329-335

15 Nuwal P, Dixit R, Dargar P, George J. Pleural effusion as the initial manifestation of chronic myeloid leukemia: Report of a case with clinical and cytologic correlation. J Cytol 2012;29(02):152-154 